\title{
Hey1 functions as a positive regulator of odontogenic differentiation in odontoblast-lineage cells
}

\author{
XIAO YIN ${ }^{1,2 *}$, ZHAOBIN ZENG $^{3 *}$, JINLIANG XING $^{4}$, ANSHENG ZHANG $^{1}$, WENKAI JIANG ${ }^{1}$, \\ WEI WANG ${ }^{1}$, HANTANG SUN ${ }^{1,5}$ and LONGXING NI ${ }^{1}$
}

\begin{abstract}
${ }^{1}$ State Key Laboratory of Military Stomatology and National Clinical Research Center for Oral Diseases and Shaanxi Key Laboratory of Oral Diseases, Department of Operative Dentistry and Endodontics, School of Stomatology, The Fourth Military Medical University, Xi'an, Shaanxi 710032; ${ }^{2}$ Department of Stomatology, Central Hospital of Xi'an,

Xi'an, Shaanxi 710003; ${ }^{3}$ Department of Stomatology, General Hospital of Shenyang Military Area Command, Shenyang, Liaoning 110015; ${ }^{4}$ Preclinical Medical Teaching Experimental Center, School of Basic Medical Sciences, The Fourth Military Medical University, Xi'an, Shaanxi 710032; ${ }^{5}$ Department of Operative Dentistry and Endodontics, Nantong Stomatological Hospital, Nantong, Jiangsu 226001, P.R. China
\end{abstract}

Received March 11, 2016; Accepted October 31, 2017

DOI: $10.3892 /$ ijmm.2017.3254

\begin{abstract}
Substantial evidence has indicated that Notch and bone morphogenetic protein (BMP) signaling may regulate odontoblastic differentiation. Hairy/enhancer-of-split related with YRPW motif 1 (Hey1), a downstream target gene of Notch and BMP signaling, is expressed in dental pulp tissues and has been demonstrated to be responsible for osteoblast mineralization. The aim of this study was to investigate the effects of Heyl on odontoblast differentiation. The results of the study demonstrated that Heyl expression in odontoblast-lineage cells (OLCs) was upregulated by stimulation of osteoblastic/odontoblastic differentiation medium containing ascorbic acid, $\beta$-glycerol phosphate and dexamethasone. Furthermore, stable Heyl-overexpressing cells expressed higher levels of dentin sialophosphoprotein (DSPP) and exhibited higher mineralization capabilities following stimulation by differentiation medium. Furthermore, RNA interference-mediated knockdown of Heyl downregulated the expression levels of DSPP in OLCs stimulated by differentiation medium. Taken together, the findings indicate that Heyl may be a positive regulator of odontoblastic
\end{abstract}

Correspondence to: Professor Longxing Ni or Professor Hantang Sun, State Key Laboratory of Military Stomatology and National Clinical Research Center for Oral Diseases and Shaanxi Key Laboratory of Oral Diseases, Department of Operative Dentistry and Endodontics, School of Stomatology, The Fourth Military Medical University, 145 Western Changle Road, Xi'an, Shaanxi 710032, P.R. China

E-mail: longxing_ni@hotmail.com

E-mail: hantang@fmmu.edu.cn

*Contributed equally

Key words: hairy/enhancer-of-split related with YRPW motif 1, odontoblast, cell differentiation, dentin sialophosphoprotein, Notch differentiation. The present study broadens the understanding of odontoblast differentiation and biomineralization.

\section{Introduction}

Odontoblasts are neural crest-derived, highly differentiated cells aligned in a single layer at the periphery of the dental pulp. The main function of these cells is to form dentin, the largest part of the hard tissue in teeth. Following differentiation from dental papilla mesenchymal cells, functional odontoblasts synthesize and secrete collagenous and non-collagenous matrix proteins that are essential for mineralized dentin formation (1).

The differentiation of odontoblasts is a complex process regulated by reciprocal epithelium-mesenchyme interactions. A number of signaling factors are reported to be involved in this process, including Notch, bone morphogenetic protein (BMP), Wnt and transforming growth factor- $\beta$ (TGF- $\beta$ ). Notch signaling is an evolutionarily conserved pathway that is responsible for the control of cell fate through local cell-cell interactions (2). It has been well documented that during tooth development, Notch receptors and ligands are expressed in dental epithelium, dental papilla mesenchyme, ameloblast or odontoblast at different stages of tooth germ development $(3,4)$. Additionally, in the pulp of injured teeth, the expression of Notch receptors and the Delta-1 ligand is significantly upregulated $(5,6)$. These results suggest that Notch signaling is involved in primary and reparative dentinogenesis. Further evidence has demonstrated that Notch signaling has a critical role in dental pulp stem cell (DPSC) differentiation into odontoblasts in vitro $(7,8)$. BMP signaling is also a potent regulator of odontoblast differentiation. As one of the strongest signals stimulating biomineralization, BMP-2 has been identified to be required for odontoblast differentiation in vivo and in vitro (9-11). Additionally, in vitro studies have demonstrated that BMP-2 gene transfection enhances the odontogenic differentiation of DPSC and stem cells from apical papilla $(12,13)$. 
Hairy/enhancer-of-split related with YRPW motif 1 (Hey1), also known as CHF2, HRT1, Herp2 or Hesr1, is a member of the basic helix-loop-helix family. Heyl was first characterized as a downstream effector of canonical Notch signaling (14), and further investigations indicated that Heyl was also induced by TGF- $\beta$ /BMP signaling independently of Notch $(15,16)$. Numerous studies have demonstrated that Heyl is responsible for the development of various tissues, including bone, nerve, heart, muscle and vascular tissues (17-21). Our preliminary study demonstrated that Heyl was expressed in dental pulp tissues and may affect dentin sialophosphoprotein (DSPP) expression during odontogenesis (22). In addition, substantial evidence has demonstrated the regulatory roles of Heyl in mineralization $(23,24)$. However, it remains unclear whether Heyl regulates odontoblastic differentiation.

In this study, the effects of Heyl on the differentiation of odontoblasts were investigated in an odontoblast-lineage cell line (OLC) $(25,26)$. The expression of Heyl in OLCs was first observed during odontogenic differentiation. Subsequently, a plasmid encoding the full-length sequence of Heyl or Heyl-silencing short hairpin RNA (shRNA) were transfected into OLCs to compare the differentiation and mineralization capabilities of cells expressing different levels of Heyl. The findings suggested that Heyl has an important role in odontoblastic differentiation.

\section{Materials and methods}

Cell culture and differentiation induction. OLC cell line was provided by Professor S. Arany (Department of Biochemistry, Akita University School of Medicine, Akita, Japan). It is a murine spontaneously immortalized cell line which was developed by Arany et al (25). OLCs were cultured in $\alpha$-minimum essential medium ( $\alpha$-MEM) supplemented with $10 \%$ fetal bovine serum (FBS; both from Hyclone; GE Healthcare Life Sciences, Logan, UT, USA) at $37^{\circ} \mathrm{C}$ in a humidified atmosphere of $5 \% \mathrm{CO}_{2}$ and $95 \%$ air. For differentiation induction, cells were plated at a density of $2 \times 10^{4} \mathrm{cell} / \mathrm{s} / \mathrm{cm}^{2}$ in 6 -well plates and were cultured until they reached $80 \%$ confluence. Cells were in serum-free $\alpha$-MEM for $24 \mathrm{~h}$ to be synchronized, then in osteoblastic/odontoblastic differentiation medium [ $\alpha$-MEM supplemented with $10 \%$ FBS, $50 \mu \mathrm{g} / \mathrm{ml}$ ascorbic acid (AA), $10 \mathrm{mM} \beta$-glycerol phosphate $(\beta-G P)$ and $10^{-8} \mathrm{M}$ dexamethasone (DEX)] $(27,28)$.

Establishment of stable Heyl-overexpressing cell lines. The plasmid encoding the full-length sequence of mouse Heyl with a C-terminal His-tag, obtained from Dr Nobuyuki Kawashima (Department of Endodontics and Dental Pulp Biology, Tokyo Medical and Dental University, Tokyo, Japan), was constructed using eukaryotic expression vector pEF-Dest51 (Invitrogen; Thermo Fisher Scientific, Inc., Waltham, MA, USA) and was named pEF-Heyl. Following confirmation by DNA sequencing, pEF-Heyl was transfected into OLCs using Lipofectamine ${ }^{\circledR} 2000$ reagent (Invitrogen; Thermo Fisher Scientific, Inc.) according to the manufacturer's instructions. Following $24 \mathrm{~h}$ of transfection, the cells were subcultured at 1:12 for another $24 \mathrm{~h}$ and selected in growth medium containing $4 \mu \mathrm{g} / \mathrm{ml}$ blasticidin (Invitrogen; Thermo Fisher Scientific, Inc.). Single cell isolation was performed using 96 -well plates. These single cell clones were amplified in blasticidin selection medium and then processed for selection by reverse-transcription polymerase chain reaction (RT-PCR) and western blot analyses. Empty pEF-Dest51 vector was transfected into OLCs as a mock negative control.

Construction and transient transfection of shRNA expression vectors targeting Heyl. A mouse Heyl-targeting sequence (5'-TGA AGGACTCGATGCCTCCGA-3') was designed using Invitrogen's online RNAi designer and was verified using BLAST to avoid off-target gene silencing. Two pairs of oligonucleotides coding shRNA, one pair containing mouse Heyl-targeting sequence and the other containing a scrambled sequence (5'-GTT CTC CGA ACG TGT CAC GT-3') with no significant similarity to any mouse gene sequences, were synthesized. Pairs of oligonucleotides were annealed and inserted into the shRNA expression vector pGPU6/GFP/Neo (Shanghai GenePharma Co., Ltd., Shanghai, China). Transient transfections into OLCs were performed using Lipofectamine ${ }^{\circledR} 2000$ reagent (Invitrogen; Thermo Fisher Scientific, Inc.) to yield OLC/Heyl-knockdown (KD) and OLC/pGP-Mock.

$R N A$ preparation and $R T$-quantitative $P C R(R T-q P C R)$. Total RNA was extracted from OLCs using TRIzol reagent (Thermo Fisher Scientific, Inc.) and was quantified by spectrophotometry using a NanoDrop 2000c spectrophotometer (Thermo Fisher Scientific, Inc., Wilmington, DE, USA). Total RNA $(1 \mu \mathrm{g})$ was reverse transcribed into cDNA using a First Strand cDNA Synthesis kit (Thermo Fisher Scientific, Inc.) according to the manufacturer's guidelines. qPCR was performed on $1 \mu 1$ of cDNA in a $20 \mu 1$ reaction with SYBR Premix Ex Taq (Takara Biotechnology Co., Ltd., Dalian, China) using the Bio-Rad CFX96 real-time PCR detection system (Bio-Rad Laboratories, Inc., Hercules, CA, USA). The sense and antisense primers were as follows: 5'-CGACGAGACCGAATC AATAAC-3' and 5'-CAAACTCCGATAGTCCATAGCC-3' for Hey1; 5'-AGCATCAAGAATAGCACCAACC-3' and 5'-CCC ATCAGTATCATCCAAACCT-3' for DSPP; 5'-GACCCCTTC ATTGACCTCA-3' and 5'-GCTCCTGGAAGATGGTGA-3' for GAPDH. The protocol for the qPCR reactions consisted of an initial denaturation step $\left(95^{\circ} \mathrm{C}\right.$ for $\left.3 \mathrm{~min}\right)$, followed by 45 cycles of denaturation $\left(95^{\circ} \mathrm{C}\right.$ for $\left.10 \mathrm{sec}\right)$, annealing $\left(55^{\circ} \mathrm{C}\right.$ for $10 \mathrm{sec})$, and extension $\left(72^{\circ} \mathrm{C}\right.$ for $\left.20 \mathrm{sec}\right)$. GAPDH was used as the housekeeping gene for template normalization. The relative expression level of mRNA was calculated using $2^{-\Delta \Delta C q}$ analysis (29). All RT-qPCR reactions were performed in triplicate.

Western blot analysis. The cells were washed with cold phosphate-buffered saline and lysed on ice using radioimmunoprecipitation assay lysis buffer (Thermo Fisher Scientific, Inc.) containing $1 \mathrm{mM}$ phenylmethylsulfonyl fluoride. The cell lysates were centrifuged at $4^{\circ} \mathrm{C}$ for $20 \mathrm{~min}$ at $13,500 \mathrm{x} \mathrm{g}$, and the total protein content of the supernatant was collected. Protein concentration was determined using a bicinchoninic acid protein assay kit (Thermo Fisher Scientific, Inc.). The protein samples were mixed with $5 \mathrm{X}$ loading buffer and then were boiled for denaturation. Protein extracts $(30 \mu \mathrm{g})$ from each sample were subjected to $8 \%$ SDS-PAGE and were transferred to polyvinylidene difluoride membranes (EMD Millipore, Billerica, MA, USA). The membranes were 
A

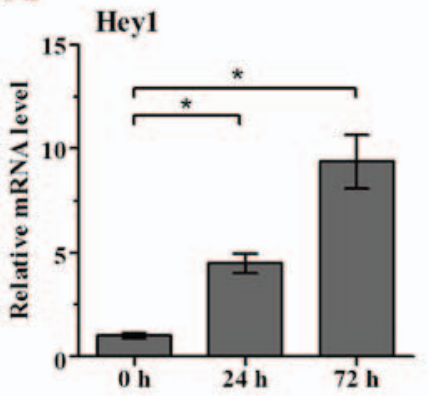

B

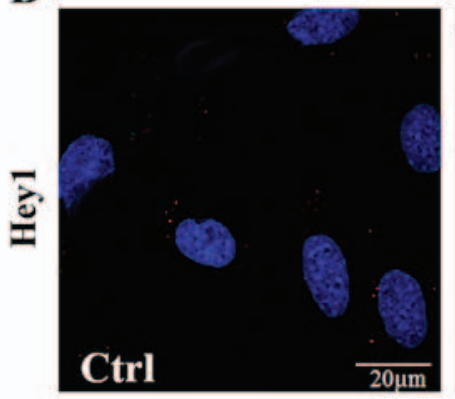

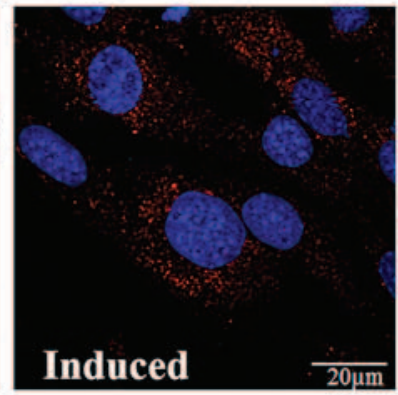

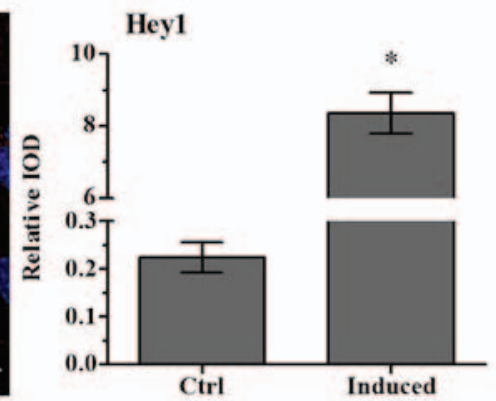

Figure 1. Odontoblastic differentiation medium induced an increase in Heyl expression in OLCs. (A) Hey1 mRNA levels in OLCs cultured in differentiation medium were evaluated by reverse transcription-quantitative polymerase chain reaction on the 1st and 3rd days after induction. (B) Hey1 expression in protein levels in OLCs cultured by normal or differentiation mediums for 5 days was determined by immunofluorescence staining. Data are presented as the mean \pm standard deviation. ${ }^{*} \mathrm{P}<0.05$. Hey1, hairy/enhancer-of-split related with YRPW motif 1; OLCs, odontoblast-lineage cells; IOD, integrated optical density.

blocked in $5 \% \mathrm{BSA}$ for $2 \mathrm{~h}$ at $37^{\circ} \mathrm{C}$ and were incubated with rabbit anti-His-probe (cat. no. sc-803; 1:500), anti-dentin sialoprotein (DSP; cat. no. sc-33587; 1:200) both from Santa Cruz Biotechnology, Inc. (Dallas, TX, USA) or anti- $\beta$-actin (cat. no. ab8227; 1:2,000; Abcam, Cambridge, UK) primary antibodies overnight at $4^{\circ} \mathrm{C}$. The membranes were then rinsed in TBS-Tween and incubated with an HRP-conjugated goat anti-rabbit secondary antibody (cat. no. AP307P, 1:5,000; EMD Millipore) for $1 \mathrm{~h}$, and then detected using an enhanced chemiluminescence system (GE ImageQuant 350; GE Healthcare, Piscataway, NJ, USA). Semi-quantitative analyses of the bands were performed using Image-Pro Plus 6.0 software (Media Cybernetics, Inc., Rockville, MD, USA).

Immunofluorescence staining. OLCs in $35 \mathrm{~mm}$ glass bottom dishes were fixed with $4 \%$ paraformaldehyde for $30 \mathrm{~min}$ at room temperature, permeabilized in $0.1 \%$ Triton $\mathrm{X}-100$ for 5 min, and blocked with $2 \%$ goat serum (cat. no. C0265; Beyotime Biotechnology, Shanghai, China) at $37^{\circ} \mathrm{C}$ for $1 \mathrm{~h}$. Cells were then incubated with rabbit anti-Heyl (cat. no. ab22614; 1:50; Abcam) or anti-DSP (cat. no. sc-33587; 1:50; Santa Cruz Biotechnology, Inc.) primary antibody at $4^{\circ} \mathrm{C}$ overnight. Finally, cells were incubated with Alexa Fluor 594-conjugated goat anti-rabbit IgG secondary antibody (cat. no. A11037; 1:400; Invitrogen; Thermo Fisher Scientific, Inc.) at $37^{\circ} \mathrm{C}$ for $1 \mathrm{~h}$, and nuclei were stained with $2 \mu \mathrm{g} / \mathrm{ml}$ DAPI (cat. no. C1002; Beyotime Biotechnology) for $5 \mathrm{~min}$ at room temperature. Fluorescence was examined using a FV1000 confocal laser scanning microscope (Olympus Corporation, Tokyo, Japan). Fluorescence intensity was determined with Image-Pro Plus 6.0 software (Media Cybernetics, Inc.).

Mineralization assay. Mock OLCs and stable Heyl-overexpressing cells were plated in 6-well plates and cultured by osteoblastic/odontoblastic differentiation medium, respectively. Media were collected every 4 days for determination of alkaline phosphatase (ALP) activity using an Alkaline Phosphatase assay kit (Jiancheng Bioengineering Institute, Nanjing, China) as described in a previous study (30). In brief, $20 \mu \mathrm{l}$ of cell culture medium mixed with $1 \mathrm{ml}$ of reaction solution containing $18 \mathrm{mM}$ 4-nitrophenyl phosphate and $0.5 \mathrm{M}$ 2-amino-2-methyl-1-propanol was incubated in the dark for
$15 \mathrm{~min}$ at $37^{\circ} \mathrm{C}$. ALP activity was quantified by measuring the absorbance values of the reaction solution at $405 \mathrm{~nm}$ using an absorbance microplate reader (BioTek Instruments, Inc., Winooski, VT, USA). After culture for 32 days, cells were rinsed with distilled water and fixed with $4 \%$ paraformaldehyde for $30 \mathrm{~min}$ at room temperature. Mineralized deposits were then stained with $40 \mathrm{mM}$ alizarin red S (cat. no. A5533; Sigma-Aldrich, St. Louis, MO, USA) for $30 \mathrm{~min}$ at room temperature. The excess dye was removed by washing 3 times with distilled water. Red stain of mineralized deposits was observed by a light microscope (Olympus Corporation).

Statistical analysis. All values presented are expressed as the mean \pm standard deviation. One-way analysis of variance was used to analyze the differences between the groups. The differences between groups were detected with post hoc Student-Newman-Keuls tests. $\mathrm{P}<0.05$ was considered to indicate a statistically significant difference.

\section{Results}

Odontoblastic differentiation medium induces an increase in Heyl expression. Hey1 mRNA levels in the OLCs were significantly increased on the day 1 and 3 of culture in osteoblastic/odontoblastic differentiation medium containing AA, $\beta$-GP and DEX (Fig. 1A). Additionally, immunofluorescence staining was performed to evaluate the protein levels of Heyl during differentiation induction. There was almost no positive staining in the untreated OLCs. However, the expression of Heyl protein was observed in OLCs following stimulation with differentiation medium for 5 days (Fig. 1B).

Stable Heyl-overexpressing cells line was established. To investigate the effects of Heyl on the differentiation of odontoblasts, pEF-Hey1 and the control vector pEF-Dest51 were transfected into OLCs. Following pEF-Heyl transfection, four single cell clones that were resistant to blasticidin were amplified. RT-PCR demonstrated that Hey1 mRNA expression was barely detected in normal OLCs (untransfected) or mock-transfected cells (transfected with empty pEF-Dest51), whereas the four single cell clones transfected with pEF-Heyl expressed much higher levels of Heyl mRNA (Fig. 2A). Western blot 
A
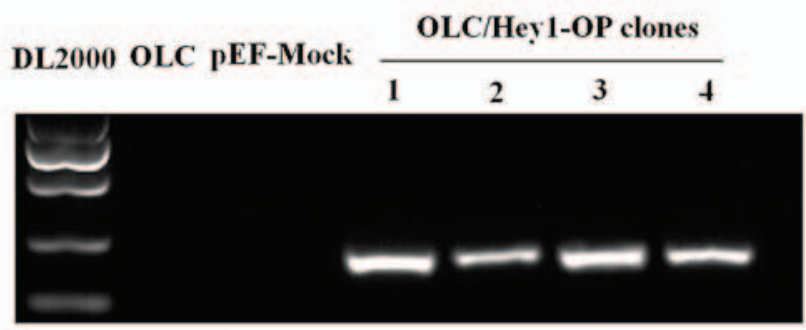

B
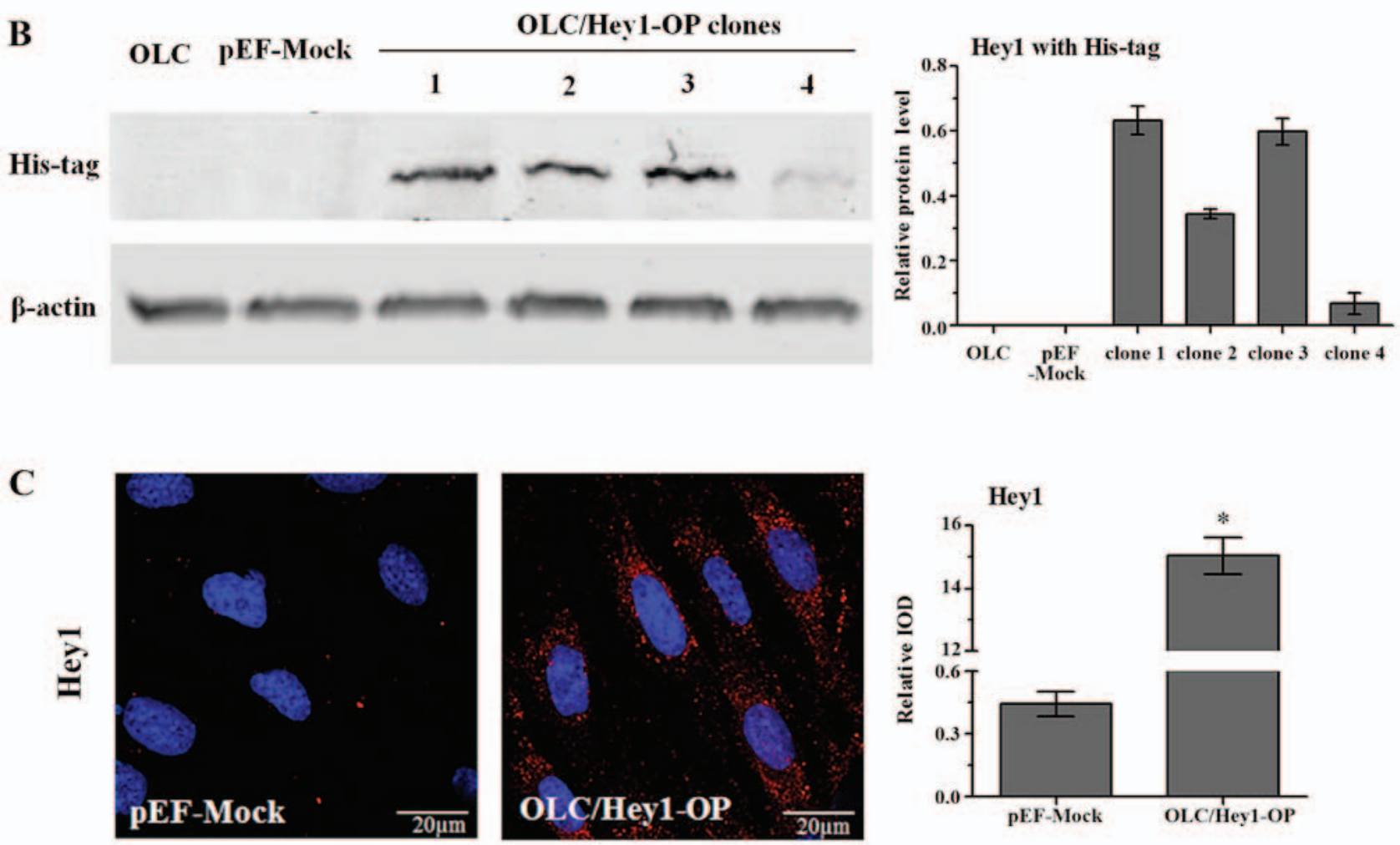

Figure 2. Heyl-overexpressing cell line OLC/Hey1-OP was established. (A) pEF-Heyl and empty pEF-Dest51 were individually transfected into OLCs, and four resistant cell clones were isolated. Four Heyl-OP clones expressed much higher levels of Heyl mRNA than the OLCs and mock cells. (B) Among the four clones, clone one synthesized the highest levels of Heyl protein. (C) Immunofluorescence staining for Heyl protein in OLC/pEF-Mock and OLC/Heyl-OP. Data are presented as the mean \pm standard deviation. ${ }^{*} \mathrm{P}<0.05$. Heyl, stable hairy/enhancer-of-split related with YRPW motif 1 ; OLC, odontoblast-lineage cell; OP, overexpression.

analysis with an anti-His-tag antibody was performed to verify the effectiveness of the plasmid to induce Heyl overexpression. Among the four clones, clone one synthesized the highest levels of Heyl protein (Fig. 2B) and was designated as OLC/Heyl-OP for further investigations. Immunofluorescence staining with an anti-Heyl antibody further confirmed that the OLC/Heyl-OP cell line expressed a higher level of Heyl protein compared with mock transfection cells (Fig. 2C).

Stable Heyl-overexpressing cells line exhibit increased differentiation capabilities. The differentiation capabilities between the stable Heyl-overexpressing and mock cell lines were compared. The results of RT-qPCR demonstrated that overexpressing Heyl alone did not affect the mRNA levels of DSPP, the odontoblastic differentiation marker, in OLCs $(\mathrm{P}>0.1)$. However, following culture in differentiation medium for 3 days, OLC/Heyl-OP expressed a significantly higher mRNA level of DSPP than the mock cells (Fig. 3A). Since DSPP is a large protein that can be specifically cleaved into two fractions, DSP and dentin phosphoprotein, an antibody against the DSP portion of DSPP was used to detect protein expression of the full-length DSPP. The results of western blot analysis and immunofluorescence staining further revealed that Heyl overexpression increased DSPP protein expression in OLCs when the cells were induced by differentiation medium for 7 days (Fig. 3B and C). Furthermore, when cultured in differentiation medium, the ALP activities of the OLC/Heyl-OP cells were much higher than that of the mock cells (Fig. 4A). Alizarin red S staining revealed that the OLC/Hey1-OP cells formed more and larger mineralized nodules than the mock cells (Fig. 4B).

Transient Heyl knockdown inhibited OLC differentiation. To further determine whether Heyl is critical for odontoblastic differentiation, a Heyl-targeting shRNA expression vector was constructed, and transient transfection into OLCs was performed. To evaluate the efficiency of the RNA interference in silencing Hey1 expression, the mRNA and protein expression levels of Heyl in transfected OLCs and mock cells were measured using 

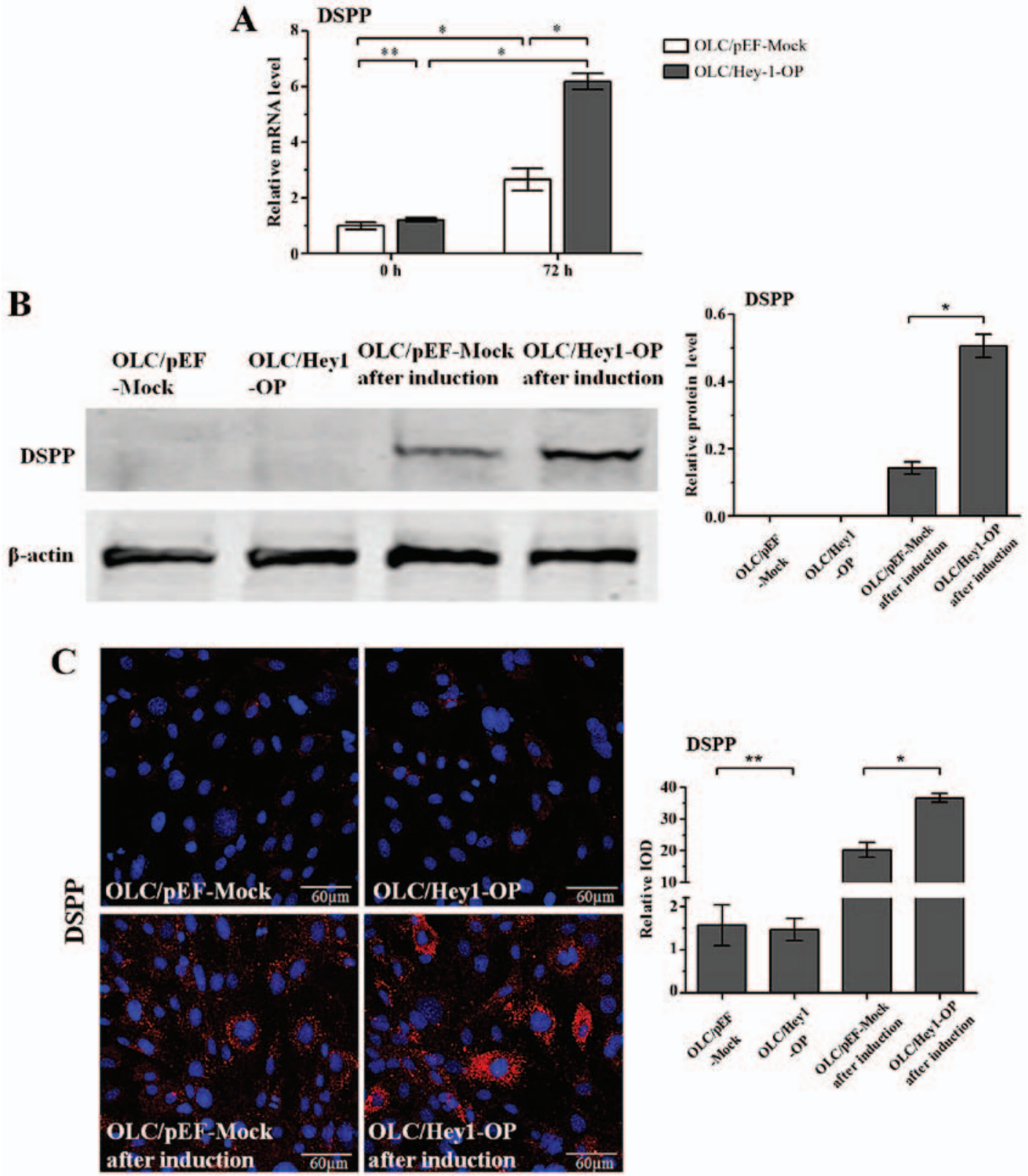

Figure 3. Stable Heyl-overexpressing cell line expressed a higher level of DSPP following culture in differentiation medium. (A) mRNA levels of DSPP gene in OLC/pEF-Mock and OLC/Heyl-OP cultured by differentiation medium for 3 days. Protein levels of DSPP in OLC/pEF-Mock and OLC/Heyl-OP cultured by differentiation medium for 7 days, determined by (B) western blotting and (C) immunofluorescence staining. Data are presented as the mean \pm standard deviation. "P<0.05 and ${ }^{* *} \mathrm{P}>0.1$. DSPP, dentin sialophosphoprotein; Hey1, hairy/enhancer-of-split related with YRPW motif 1; OP, overexpression; OLC, odontoblast-lineage cell; IOD, integrated optical density.

A

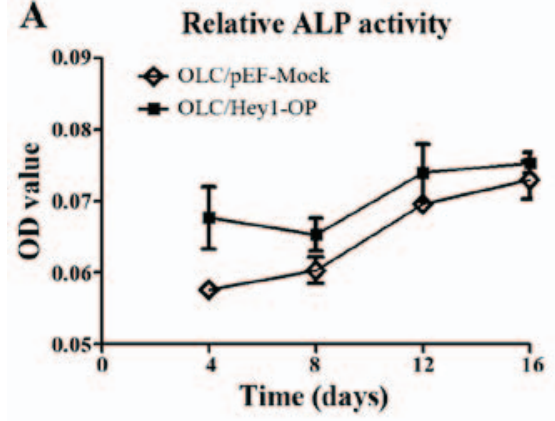

B

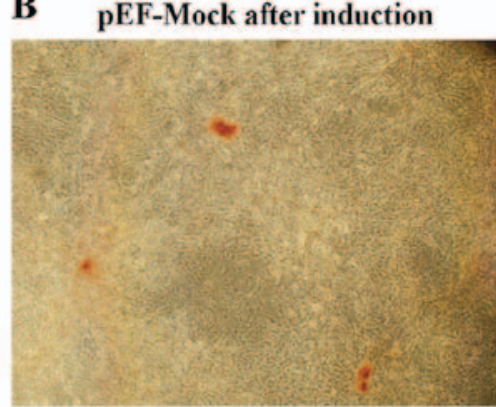

OLC/Hey1-OP after induction

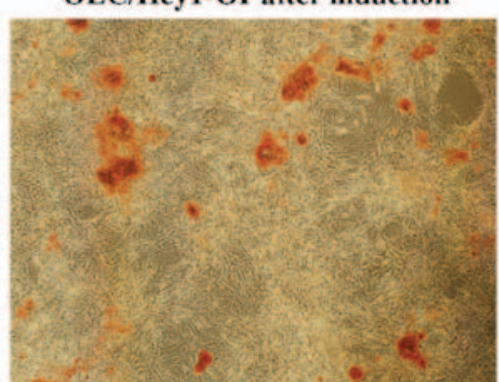

Figure 4. Hey1 overexpression promoted ALP activity and OLC mineralization. (A) The ALP activities secreted into the medium by OLC/pEF-Mock and OLC/Heyl-OP during differentiation induction. (B) Alizarin red S staining of OLC/pEF-Mock and OLC/Heyl-OP cultured in differentiation medium for 32 days. Heyl, hairy/enhancer-of-split related with YRPW motif 1; OP, overexpression; ALP, alkaline phosphatase; OLC, odontoblast-lineage cell; OD, optical density. 
A

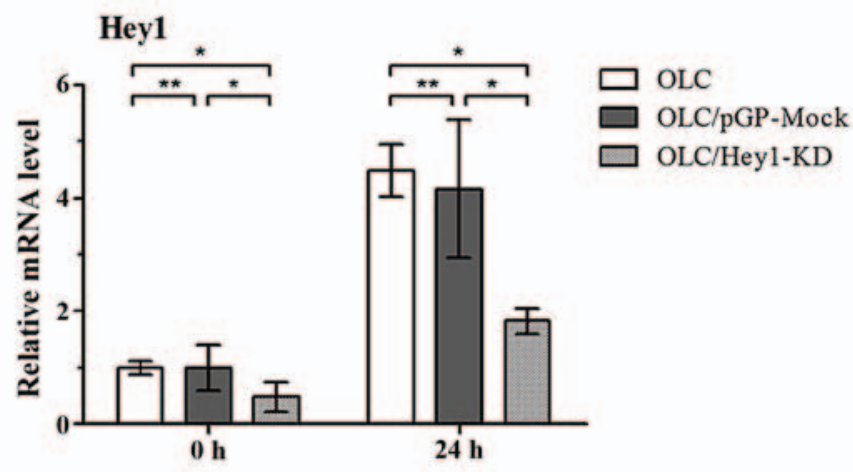

B
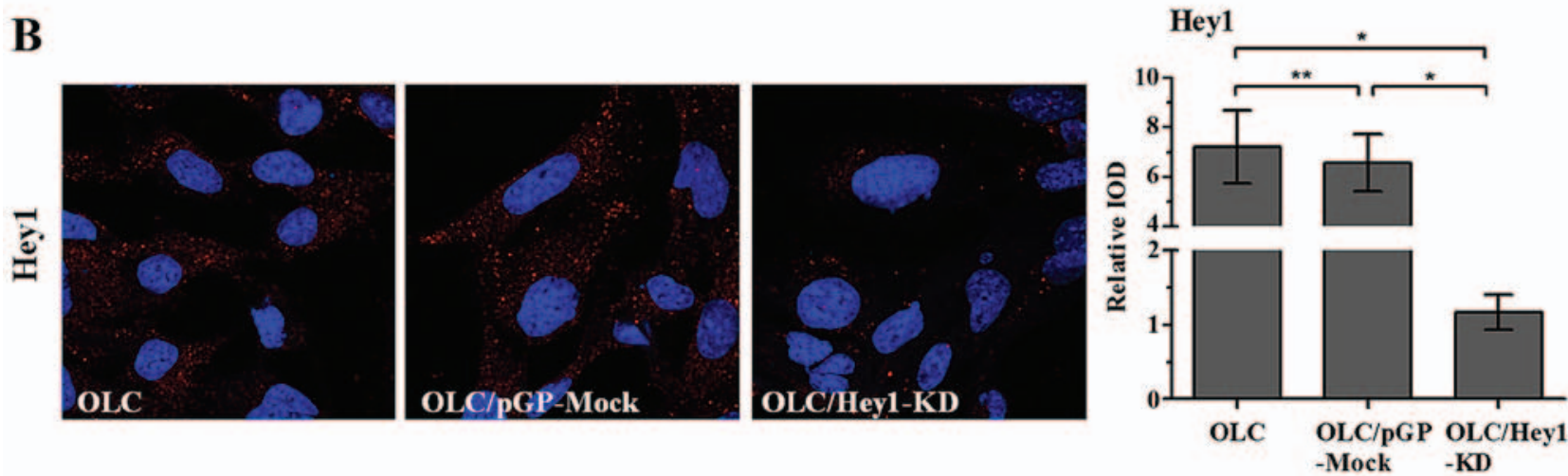

Figure 5. Short hairpin RNA-mediated inhibition of Heyl expression. (A) mRNA levels of Hey1 in OLCs, OLC/pGP-Mock and OLC/Hey1-KD cultured in differentiation medium for $24 \mathrm{~h}$. (B) Immunofluorescence staining for Hey1 protein in OLCs, OLC/pGP-Mock and OLC/Heyl-KD cultured in differentiation medium for 5 days. Data are presented as the mean \pm standard deviation. ${ }^{*} \mathrm{P}<0.05$ and ${ }^{* *} \mathrm{P}>0.1$. Hey1, hairy/enhancer-of-split related with YRPW motif 1 ; OLC, odontoblast-lineage cells; KD, knockdown; IOD, integrated optical density.

RT-qPCR and immunofluorescence staining, respectively, following stimulation in differentiation medium. OLC/Heyl-KD cells expressed lower levels of Heyl mRNA and protein than OLC/pGP-Mock cells ( $\mathrm{P}<0.05$; Fig. 5). Further investigations revealed that DSPP expression was decreased in OLC/Heyl-KD cells compared with the mock cells following differentiation induction (Fig. 6). Following culturing in differentiation medium for 3 days, the mRNA level of DSPP in OLC/Heyl-KD cells was significantly lower than OLC/pGP-Mock cells (Fig. 6A). Furthermore, after culture in differentiation medium for 7 days, DSPP protein expression in OLC/Heyl-KD cells was significantly lower than in OLC/pGP-Mock cells (Fig. 6B and C).

\section{Discussion}

Differentiation of the progenitor cells derived from dental pulp tissue into odontoblasts has a vital role in the dentinal regeneration process. Elucidating the underlying mechanisms will facilitate the development of therapeutic approaches for injured dentin-pulp complex. Numerous methods have been used to induce odontoblast differentiation in vitro. The application of osteoblastic/ odontoblastic differentiation medium (containing AA, $\beta$-GP and DEX) has been demonstrated to be an effective method and has been adopted in numerous studies $(7,8,27,28,31-33)$. Previous in vitro studies have demonstrated the involvement of Notch and BMP signaling in odontoblast differentiation induced by AA + $\beta$-GP + DEX $(7,8,32,33)$. However, the underlying mechanisms remain unclear. The present study revealed that Heyl expression in odontoblast-lineage cells was significantly upregulated by $\mathrm{AA}+\beta-\mathrm{GP}+\mathrm{DEX}$ stimulation, suggesting that Heyl may be involved in their differentiation. Because Hey1 has been reported to regulate osteoblast differentiation and matrix mineralization $(23,24,30)$, it is assumed that Heyl may have an important role in odontoblast differentiation. To verify this hypothesis, Heyl overexpression and knockdown models were established in vitro to investigate the effects of Heyl on odontoblast differentiation.

DSPP, a non-collagenous protein that is predominantly expressed in odontoblasts or dentin, was demonstrated to be critical for dentin mineralization. DSPP is synthesized and secreted by differentiated odontoblasts and is regarded as a marker of odontogenic differentiation $(34,35)$. In the present study, OLCs cultured in differentiation medium exhibited increased expression of DSPP, a result is consistent with previous in vitro studies. Furthermore, the results revealed that overexpression of Heyl did not directly upregulate DSPP expression in OLCs but enhanced the upregulatory effect of $\mathrm{AA}+\beta-\mathrm{GP}+\mathrm{DEX}$ stimulation on DSPP expression. Previous studies have demonstrated that Heyl not only regulated downstream targets as a transcriptional repressor, but also functioned through interaction with other transcription factors $(36,37)$. Similarly, it has been shown that Hey2 overexpression alone is not sufficient to induce strong changes in downstream gene expression, but needs additional cofactors (38). Therefore, according to the results of the present study, Heyl may regulate DSPP expression indirectly by interacting with other cofactors activated in the process of odontoblastic differentiation; this requires further investigations. 
A

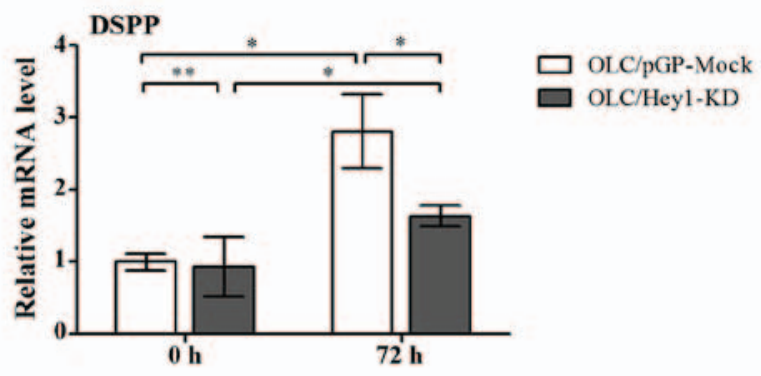

B
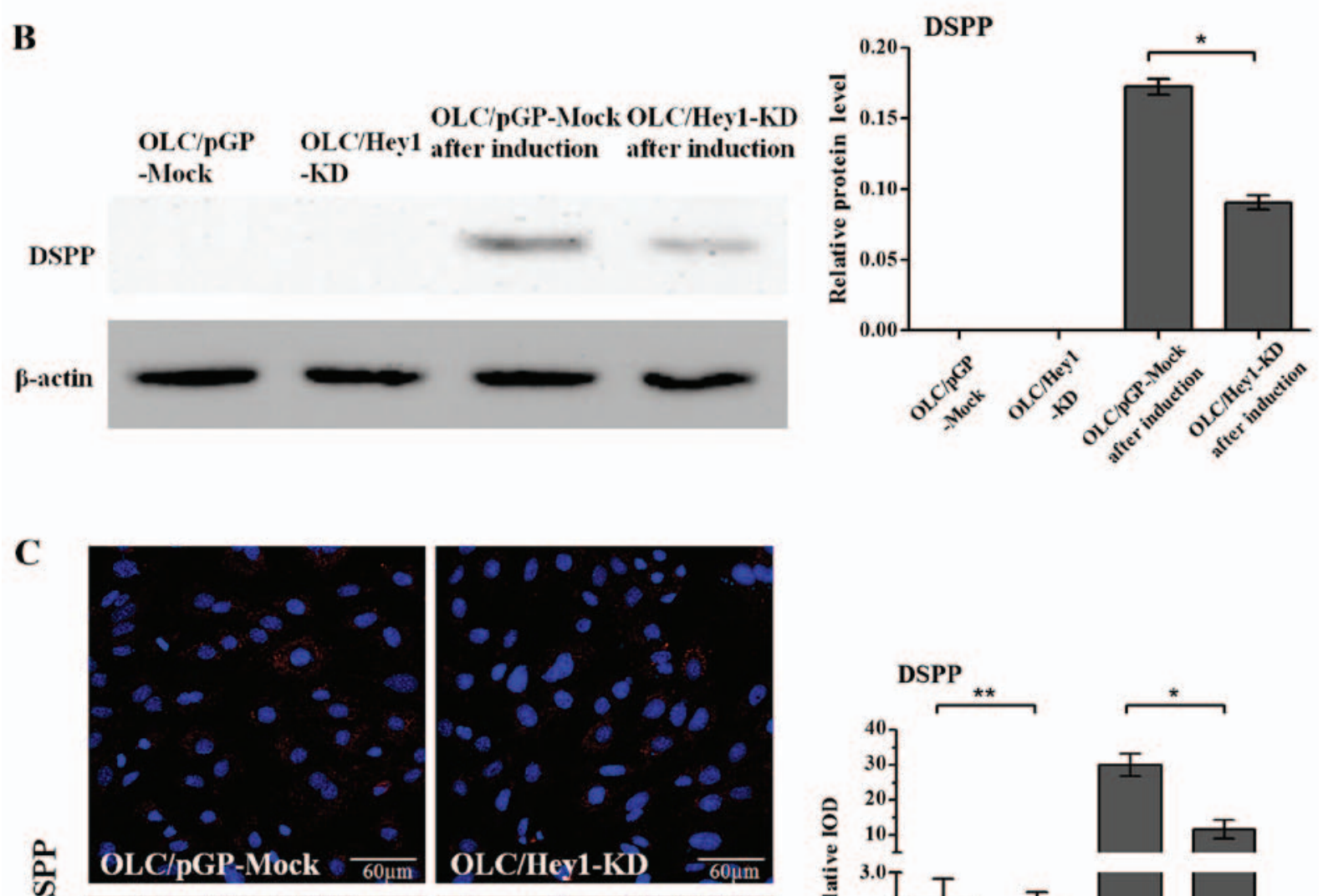

OLC/pGP

OLC/pGP-Mock OLC/Hey1-KD -Mock -KD

DSPP
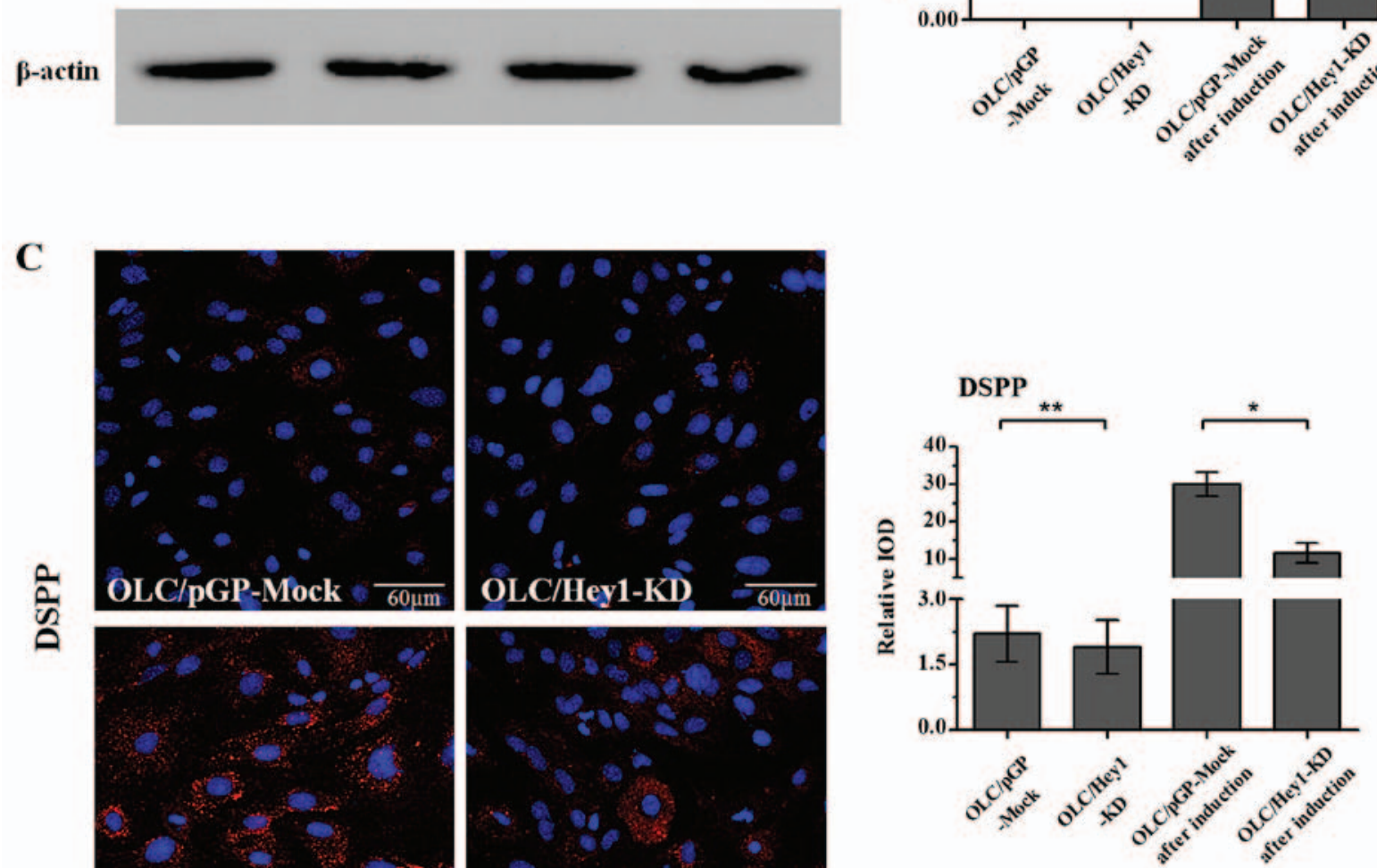

Figure 6. Hey1 KD diminished DSPP expression induced by differentiation medium. (A) mRNA levels of DSPP gene in OLC/pGP-Mock and OLC/ Heyl-KD cultured in differentiation medium for 3 days. Protein levels of DSPP in OLC/pGP-Mock and OLC/Heyl-KD cultured in differentiation medium for 7 days as determined by (B) western blotting and (C) immunofluorescence staining. Data are presented as the mean $\pm \mathrm{SD}$. ${ }^{*} \mathrm{P}<0.05$ and ${ }^{* *} \mathrm{P}>0.1$. Heyl, hairy/enhancer-of-split related with YRPW motif 1; DSPP, dentin sialophosphoprotein; OLC, odontoblast-lineage cell; KD knockdown; IOD, integrated optical density.

OLCs in which Heyl was exogenously overexpressed exhibited upregulation of ALP activity and increased nodule formation. These results indicate that Heyl may be a positive regulator of odontoblast cell mineralization. These findings are consistent with previous studies showing that Heyl enhanced osteogenic differentiation and mineralization of mesenchymal stem cells induced by BMP-9 or BMP-7 $(23,39)$. However,
Zamurovic et al (24) reported that Heyl inhibited mineralization of the MC3T3 cell line. This discrepancy may result from differences in the source of cells. Certain transcription factors, such as runt related transcription factor 2 and nuclear factor erythroid 2-related factor 1, have demonstrated different effects on the differentiation of odontoblasts and osteoblasts $(40,41)$. The function of Heyl may also be dependent on cell type. 
An in vivo study demonstrated that Heyl single knockout mice exhibited no major developmental or obvious functional impairments. However, the dental phenotypes of Heyl-deficient mice have not been analyzed (42). The present in vitro study revealed that RNA interference-mediated knockdown of Heyl expression led to decreased DSPP expression compared with the control mock cells following differentiation induction, suggesting that Heyl is critical for odontoblastic differentiation. Previous in vitro studies demonstrated that knockdown of Heyl promoted myogenesis and inhibited osteogenic differentiation $(23,43)$. Taken together, Heyl may participate in the regulation of cell fate.

In conclusion, the present study demonstrated that Hey1 was involved in the differentiation of odontoblast-lineage cells. Heyl overexpression increased DSPP expression during odontoblastic differentiation and further augmented mineralization of OLCs. Additionally, knockdown of Heyl diminished DSPP expression induced by odontoblastic differentiation medium. The findings of the current study indicate that Heyl functions as a positive regulator of odontogenic differentiation. This study broadens our understanding of odontoblast differentiation and biomineralization.

\section{Acknowledgements}

This study was supported by the National Nature Science Foundation of China (grant no. 81070832) and the National Nature Science Foundation of China (grant no. 81371139).

\section{References}

1. Ruch JV, Lesot H and Bègue-Kirn C: Odontoblast differentiation. Int J Dev Biol 39: 51-68, 1995.

2. Artavanis-Tsakonas S, Rand MD and Lake RJ: Notch signaling: Cell fate control and signal integration in development. Science 284: 770-776, 1999.

3. Mitsiadis TA, Lardelli M, Lendahl U and Thesleff I: Expression of Notch 1, 2 and 3 is regulated by epithelial-mesenchymal interactions and retinoic acid in the developing mouse tooth and associated with determination of ameloblast cell fate. J Cell Biol 130: 407-418, 1995.

4. Cai X, Gong P, Huang Y and Lin Y: Notch signalling pathway in tooth development and adult dental cells. Cell Prolif 44: 495-507, 2011.

5. Løvschall H, Tummers M, Thesleff I, Füchtbauer EM and Poulsen K: Activation of the Notch signaling pathway in response to pulp capping of rat molars. Eur J Oral Sci 113: 312-317, 2005.

6. Mitsiadis TA, Fried K and Goridis C: Reactivation of Delta-Notch signaling after injury: Complementary expression patterns of ligand and receptor in dental pulp. Exp Cell Res 246: 312-318, 1999.

7. Wang X, He F, Tan Y, Tian W and Qiu S: Inhibition of Delta1 promotes differentiation of odontoblasts and inhibits proliferation of human dental pulp stem cell in vitro. Arch Oral Biol 56: 837-845, 2011.

8. Zhang C, Chang J, Sonoyama W, Shi S and Wang CY: Inhibition of human dental pulp stem cell differentiation by Notch signaling. J Dent Res 87: 250-255, 2008.

9. Yang W, Harris MA, Cui Y, Mishina Y, Harris SE and Gluhak-Heinrich J: Bmp2 is required for odontoblast differentiation and pulp vasculogenesis. J Dent Res 91: 58-64, 2012.

10. Cho YD, Yoon WJ, Woo KM, Baek JH, Park JC and Ryoo HM: The canonical BMP signaling pathway plays a crucial part in stimulation of dentin sialophosphoprotein expression by BMP-2. J Biol Chem 285: 36369-36376, 2010.

11. Washio A, Kitamura C, Morotomi T, Terashita M and Nishihara T: Possible involvement of Smad signaling pathways ininduction of odontoblastic properties in $\mathrm{KN}-3$ cells by bone morphogenetic protein-2: A growth factor to induce dentin regeneration. Int $\mathrm{J}$ Dent 2012: 258469, 2012.
12. Zhang W, Zhang X, Ling J, Liu W, Zhang X, Ma J and Zheng J: Proliferation and odontogenic differentiation of BMP2 gene transfected stem cells from human tooth apical papilla: An in vitro study. Int J Mol Med 34: 1004-1012, 2014.

13. Yang X, van der Kraan PM, van den Dolder J, Walboomers XF, Bian Z, Fan M and Jansen JA: STRO-1 selected rat dental pulp stem cells transfected with adenoviral-mediated human bone morphogenetic protein 2 gene show enhanced odontogenic differentiation. Tissue Eng 13: 2803-2812, 2007.

14. Iso T, Kedes L and Hamamori Y: HES and HERP families: Multiple effectors of the Notch signaling pathway. J Cell Physiol 194: 237-255, 2003

15. Zavadil J, Cermak L, Soto-Nieves N and Böttinger EP: Integration of TGF-beta/Smad and Jagged1/Notch signalling in epithelial-to-mesenchymal transition. EMBO J 23: 1155-1165, 2004.

16. Wöltje K, Jabs M and Fischer A: Serum induces transcription of Heyl and Hey 2 genes by Alk1 but not Notch signaling in endothelial cells. PLoS One 10: e0120547, 2015.

17. Salie R, Kneissel M, Vukevic M, Zamurovic N, Kramer I, Evans G, Gerwin N, Mueller M, Kinzel B and Susa M: Ubiquitous overexpression of Heyl transcription factor leads to osteopenia and chondrocyte hypertrophy in bone. Bone 46: 680-694, 2010

18. Sakamoto M, Hirata H, Ohtsuka T, Bessho Y and Kageyama R: The basic helix-loop-helix genes Hesr1/Hey1 and Hesr2/Hey2 regulate maintenance of neural precursor cells in the brain. J Biol Chem 278: 44808-44815, 2003.

19. Kokubo H, Tomita-Miyagawa S, Hamada Y and Saga Y: Hesrl and Hesr2 regulate atrioventricular boundary formation in the developing heart through the repression of Tbx2. Development 134: 747-755, 2007.

20. Buas MF, Kabak S and Kadesch T: The Notch effector Heyl associates with myogenic target genes to repress myogenesis. J Biol Chem 285: 1249-1258, 2010.

21. Itoh F, Itoh S, Goumans MJ, Valdimarsdottir G, Iso T, Dotto GP, Hamamori Y, Kedes L, Kato M and ten Dijke Pt P: Synergy and antagonism between Notch and BMP receptor signaling pathways in endothelial cells. EMBO J 23: 541-551, 2004.

22. Sun H, Kawashima N, Xu J, Takahashi S and Suda H: Expression of Notch signalling-related genes in normal and differentiating rat dental pulp cells. Aust Endod J 36: 54-58, 2010.

23. Sharff KA, Song WX, Luo X, Tang N, Luo J, Chen J, Bi Y, He BC, Huang $\mathrm{J}, \mathrm{Li} \mathrm{X}$, et al: Heyl basic helix-loop-helix protein plays an important role in mediating BMP9-induced osteogenic differentiation of mesenchymal progenitor cells. J Biol Chem 284: 649-659, 2009.

24. Zamurovic N, Cappellen D, Rohner D and Susa M: Coordinated activation of notch, Wnt, and transforming growth factor-beta signaling pathways in bone morphogenic protein 2-induced osteogenesis. Notch target gene Heyl inhibits mineralization and Runx2 transcriptional activity. J Biol Chem 279: 37704-37715, 2004.

25. Arany S, Nakata A, Kameda T, Koyota S, Ueno Y and Sugiyama T: Phenotype properties of a novel spontaneously immortalized odontoblast-lineage cell line. Biochem Biophys Res Commun 342: 718-724, 2006.

26. Wang H, Kawashima N, Iwata T, Xu J, Takahashi S, Sugiyama T and Suda H: Differentiation of odontoblasts is negatively regulated by MEPE via its C-terminal fragment. Biochem Biophys Res Commun 398: 406-412, 2010.

27. Han N, Zheng Y, Li R, Li X, Zhou M, Niu Y and Zhang Q: $\beta$-catenin enhances odontoblastic differentiation of dental pulp cells through activation of Runx2. PLoS One 9: e88890, 2014.

28. Salmon B, Bardet C, Khaddam M, Naji J, Coyac BR, Baroukh B, Letourneur F, Lesieur J, Decup F, Le Denmat D, et al: MEPE-derived ASARM peptide inhibits odontogenic differentiation of dental pulp stem cells and impairs mineralization in tooth models of X-linked hypophosphatemia. PLoS One 8: e56749, 2013.

29. Livak KJ and Schmittgen TD: Analysis of relative gene expression data using real-time quantitative PCR and the 2(-Delta Delta C(T)) Method. Methods 25: 402-408, 2001.

30. Zeng Z, Yin X, Zhang X, Jing D and Feng X: Cyclic stretch enhances bone morphogenetic protein-2-induced osteoblastic differentiation through the inhibition of Heyl. Int J Mol Med 36: 1273-1281, 2015.

31. Xu J, Yu B, Hong C and Wang CY: KDM6B epigenetically regulates odontogenic differentiation of dental mesenchymal stem cells. Int J Oral Sci 5: 200-205, 2013. 
32. Chen Z, Li W, Wang H, Wan C, Luo D, Deng S, Chen H and Chen S: Klf10 regulates odontoblast differentiation and mineralization via promoting expression of dentin matrix protein 1 and dentin sialophosphoprotein genes. Cell Tissue Res 363: 385-398, 2016.

33. Wang $\mathrm{X}, \mathrm{He} \mathrm{H}, \mathrm{Wu} \mathrm{X}, \mathrm{Hu} \mathrm{J}$ and Tan Y: Promotion of dentin regeneration via CCN3 modulation on Notch and BMP signaling pathways. Biomaterials 35: 2720-2729, 2014

34. Prasad M, Butler WT and Qin C: Dentin sialophosphoprotein in biomineralization. Connect Tissue Res 51: 404-417, 2010.

35. Yamakoshi Y: Dentinogenesis and dentin sialophosphoprotein (DSPP). J Oral Biosci 51: 134-142, 2009.

36. Weber D, Wiese C and Gessler M: Hey bHLH transcription factors. Curr Top Dev Biol 110: 285-315, 2014.

37. Heisig J, Weber D, Englberger E, Winkler A, Kneitz S, Sung WK, Wolf E, Eilers M, Wei CL and Gessler M: Target gene analysis by microarrays and chromatin immunoprecipitation identifies HEY proteins as highly redundant bHLH repressors. PLoS Genet 8 : e1002728, 2012.

38. Aranguren XL, Agirre X, Beerens M, Coppiello G, Uriz M Vandersmissen I, Benkheil M, Panadero J, Aguado N, Pascual-Montano A, et al: Unraveling a novel transcription factor code determining the human arterial-specific endothelial cell signature. Blood 122: 3982-3992, 2013.
39. Lavery K, Hawley S, Swain P, Rooney R, Falb D and Alaoui-Ismaili MH: New insights into BMP-7 mediated osteoblastic differentiation of primary human mesenchymal stem cells. Bone 45: 27-41, 2009.

40. Jacob A, Zhang Y and George A: Transcriptional regulation of dentin matrix protein 1 (DMP1) in odontoblasts and osteoblasts. Connect Tissue Res 55 (Suppl 1): 107-112, 2014.

41. Li S, Kong H, Yao N, Yu Q, Wang P, Lin Y, Wang J, Kuang R, Zhao X, Xu J, et al: The role of runt-related transcription factor 2 (Runx2) in the late stage of odontoblast differentiation and dentin formation. Biochem Biophys Res Commun 410: 698-704, 2011.

42. Fuke S, Minami N, Kokubo H, Yoshikawa A, Yasumatsu H, Sasagawa N, Saga Y, Tsukahara T and Ishiura S: Hesrl knockout mice exhibit behavioral alterations through the dopaminergic nervous system. J Neurosci Res 84: 1555-1563, 2006.

43. Belyea BC, Naini S, Bentley RC and Linardic CM: Inhibition of the Notch-Heyl axis blocks embryonal rhabdomyosarcoma tumorigenesis. Clin Cancer Res 17: 7324-7336, 2011.

This work is licensed under a Creative Commons

Attribution-NonCommercial-NoDerivatives 4.0 International (CC BY-NC-ND 4.0) License. 